\title{
Microwave Assisted Synthesis of Some Novel Sulphonamide bearing Pyrazolone Core Structure
}

\author{
Dipti L. Namera, Khushal M. Kapadiya, Mitesh M. Chhatrola, Umed C. Bhoya* \\ Chemical Research Laboratory, Department of Chemistry, Saurashtra University, \\ Rajkot - 360005, Gujarat, India \\ *E-mail address: drucbhoya@gmail.com
}

\begin{abstract}
We have described some novel Sulphonamide bearing pyrazoline derivatives synthesized by conventional method as well as microwave assisted method of synthesis. The reaction of 4-(3-methyl5-oxo-4,5-dihydro-1-H-pyrazol-1-yl)benzenesulphonamide with substituted benzaldehyde in the presence of Methanol as solvent and piperidine as catalyst, generated a series of substituted pyrazolone derivatives $4 \mathrm{a}-\mathrm{m}$. The structures of all synthesized compounds are well characterized by Mass spectroscopy, FT-IR, ${ }^{1} \mathrm{H}$ NMR and elemental analysis. After obtaining experimental data regarding the yield and the time taken for the synthesis by both the approaches, convenient and microwave assisted method, it was ascertained that the microwave assisted method is more suitable for synthesis of pyrazolone derivatives 4a-m.
\end{abstract}

Keywords: Pyrazolone; Arylidene; Sulphonamide

\section{INTRODUCTION}

Pyrazolone, a five-membered-ring lactam, is a derivative of pyrazole that has an additional keto $(=\mathrm{O})$ group. It has a molecular formula of $\mathrm{C}_{3} \mathrm{H}_{4} \mathrm{NO}$. The chemistry of pyrazolone began in 1883 when Knorr reported the first pyrazolone derivative. The reaction of phenyl hydrazine and ethyl acetoacetate resulted in novel structure identified in 1887 as 1phenyl-3-methyl-5-pyrazolone [6]. The Knorr pyrazole synthesis is the reaction of hydrazine with 1,3-dicarbonyl compounds to provide the pyrazole or pyrazolone ring system. The prototype molecule, antipyrine was synthesized for clinical use in 1883 . The pyrazolone nucleus has been known to exist in three tautomeric structures [7].

All these compounds are characterized by the presence of a phenyl group attached to nitrogen atom in the 1- position and a methyl group in 3- position. Phenyl group in 1position and a methyl group in 3- position seem to be essential for antipyretic activity. Several 4,4-dimethyl derivatives, as well as Pyrazole Blue and tartrazine are derived from formula II whereas from structure III several pyrazolone dyes have been derived. When pyrazolones were discovered, they were only known as non steroidal anti-inflammatory agents (or drugs) - NSAID, but in recent times, they are known to exhibit antioxidant, anticancer, antibacterial and several other pharmacological actions [6,8]. Pyrazolones are 
very important class of heterocycles due to their wide applications in pharmacological and biological activities [3,5]. Large numbers of 2-pyrazolin-5-ones have been used as therapeutics agents such as analgesics and antipyretics $[1,2,4]$.

Microbial development of resistance, as well as economic incentives, has resulted in research and development in the search for new antibiotics in order to maintain a pool of effective drugs at all time. It is important to find out newer, safer and more effective antibiotics with broad spectrum of activity. Although several antifungal agents and the azole class of drugs are currently available there is clearly a critical need for the development of new specific antimicrobial agents.

Heterocycles containing a pyrazole / pyrazolone ring system are found to exhibit a wide spectrum of biological activities, including antibacterial and antifungal activities. Due to their easier preparation and rich biological activity, pyrazolone framework plays an essential role and represents an interesting template for combinatorial and medicinal chemistry. Similarly, pyrazole derivatives have showed significant biological activities, such as anti-microbial [16], analgesic [17], anti-inflammatory [18] and anticancer [19] activities.

This gave a great impetus to the search for potential pharmacologically active drugs carrying pyrazole substituents. Increasing antibiotic resistance in microbial populations has necessitated the search for alternate cellular targets for new and existing antimicrobial agents. It is well established that small modifications in the structure of the targets are altering their biological character as well as their physiochemical properties. A detailed literature survey on antimicrobial activity of various types of compounds clearly indicates that presence of certain pharmacophore such as pyrazole in any molecule plays an important role in enhancing activity.

Keeping in view of this and in continuation of our search on biologically potent molecules [20-23], we hereby report the synthesis and antimicrobial property of some new pyrazolone derivatives Multi drug resistance is widespread with specific relevance to Gram positive and Gram negative bacteria. Infections caused by these organisms create a serious challenge to the community.

The therapeutic problem is more pronounced in patients with immuno-compromised system or those undergoing anticancer therapy substantiating the need for design and development of novel less toxic potent antimicrobial agents. Inflammation is a nonspecific immune response in which the body reacts to infection, localized irritation, free radicals, other injury or disease [24].

Antipyrine, 2,3-dimethyl-1-phenyl-3-pyrazolin-5-one, was the first pyrazolone derivative used in the management of pain and inflammation. Simultaneous administration of several drugs to treat inflammatory conditions that might be associated with some microbial and fungal infections may cause serious health problems, especially in patients with compromised liver or kidney functions.

So the discovery of a dual anti-fungal and anti-microbial agent with potential activity and fewer adverse effects not only results in a pharmaco economic agent but also leads to better patient compliance. This study was aimed at the synthesis, characterization and screening of some new pyrazolone derivatives by most convenient microwave assisted method. 


\section{EXPERIMENTAL}

All the reactions were carried out in domestic microwave synthesizer. Melting points of all the synthesized compounds have been recorded by open capillary method. Microanalyses were performed on Euro EA Elemental Analyser, at National facility for drug discovery (NFDD) center Saurashtra university Rajkot. Reaction was monitored by thin-layer chromatography (TLC) on silica gel (Merck $60 \mathrm{~F}_{254}$ ) using (3:7) Ethyl acetate: Hexane as solvent system.

The IR spectra were recorded on a Shimadzu FT-IR-8400 instrument using $\mathrm{KBr}$ pellet method. Mass spectra were recorded on Shimadzu GC-MS-QP-2010 model using Direct Injection Probe technique. ${ }^{1} \mathrm{H}$ NMR was determined in $\mathrm{CDCl}_{3} / \mathrm{DMSO}$ solution on a Bruker Ac $400 \mathrm{MHz}$ spectrometer. Commercial grade solvents and reagents were used without further purification.

\section{1. Chemistry}

4-hydrazinyl benzene sulfonamide (2) was synthesized by refluxing 4chlorosulphonamide (compound -1) with hydrazine hydrate. Obtained compound on reaction with ethyl acetoacetate by using few drops of hydrochloric acid as a catalyst gives (compound - 3). The arylidene was synthesized by refluxing (compound-3) with corresponding aldehydes in Methanol using piperidine as a catalyst for an appropriate time to give compound 4a-m different aromatic aldehydes were chosen for synthesized series of diverse pyrazolidinone derivatives.

Compounds with the long chain aldehyde (e.g. 4-(hexyloxy) benzaldehyde) and halogen substituted aldehyde and bulky group (e.g. anthraldehyde; 3,4,5-tri methoxy benzaldehyde; 2,5-dimethoxy benzaldehyde) were also synthesized. Structure of all synthesized compound in (Scheme 1) were confirmed on the basis of IR, ${ }^{1} \mathrm{H}$ NMR, mass and element analysis. The IR spectrum of compound $4 \mathrm{~b}$ showed the absorption bands at 3317 , 3088, 2976, 1672, 1101, which were due to N-H, Aromatic $=\mathrm{CH}, \mathrm{CH}_{3}$ stretch, $\mathrm{C}=\mathrm{O}, \mathrm{C}-\mathrm{O}$, and absorption band at 1159,1330 is due to the $\mathrm{S}=\mathrm{O}$ group.

${ }^{1} \mathrm{H}$ NMR spectrum of $4 \mathrm{~b}$ showed singlet at 2.35 which is due to presence of $\mathrm{CH}_{3}$ group, singlet at 3.37 due to tri methoxy group, proton resonate at 7.73 is due to presence of $\mathrm{SO}_{2} \mathrm{NH}_{2}$ group, and $8.12,8.14,8.20$ were due to the aromatic proton. Mass spectrum of compound $4 \mathrm{~b}$ gives the molecular ion peak at Mass $(\mathrm{m} / \mathrm{e}): 431\left(\mathrm{M}^{+}\right)$. Which is confirmed the molecular formula of compound $\mathrm{C}_{20} \mathrm{H}_{21} \mathrm{~N}_{3} \mathrm{O}_{6} \mathrm{~S}$. Elemental analysis indicated the $\%$ of the elements very close to the theoretical values.

\section{2. General procedure for the synthesis of new derivatives of 4-(unsubstituted phenyl- 3-methyl-5-oxo-4, 5-dihydro-1-H-pyrazol-1-yl) benzenesulphonamide}

Mixture of 4-(3-methyl-5-oxo-4,5-dihydro-1-H-pyrazol-1-yl)benzenesulphonamide $(0.01 \mathrm{M})$ and corresponding aldehyde $(0.01 \mathrm{M})$ were taken Methanol using piperidine as a catalyst which are irradiated in microwave under 400 watt for 10-15 minutes. The reaction was monitored by TLC. After completion of reaction, the reaction mixture was allowed to cool. The precipitate formed upon cooling, was filtered off, and washed with diethyl ether and dried in vacuum. Purification of The compound of premeditated series was carried out by crystallization in Methanol. 


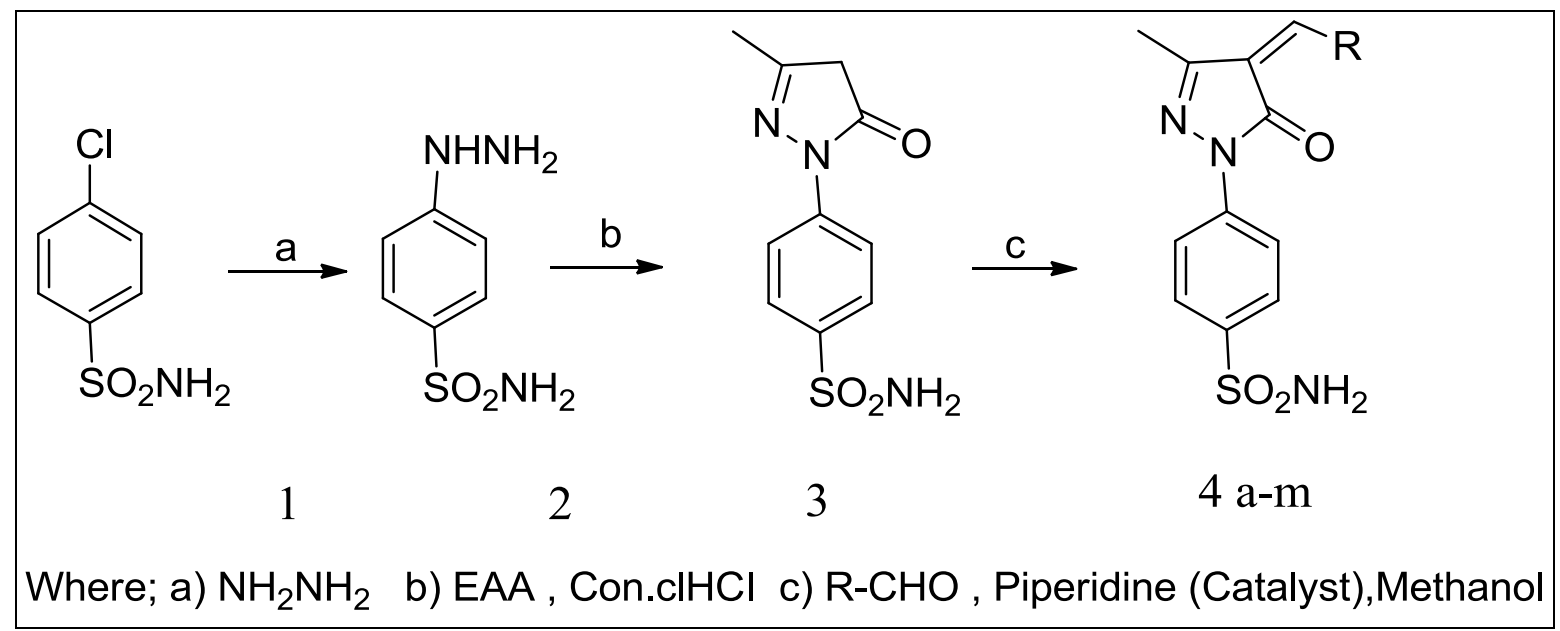

\section{3. Analytical data}

4-(4-(4-(hexyloxy) benzylidene)-3-methyl-5-oxo-4,5-dihydro-1H-pyrazol-1-yl)benzenesulfon amide; (4a)

Crystallization: Ethanol (yield $87 \%$ ), colour: yellow crystals. M.P.: $160-162{ }^{\circ} \mathrm{C}$. Anal. Calcd for $\mathrm{C}_{23} \mathrm{H}_{27} \mathrm{~N}_{3} \mathrm{O}_{4} \mathrm{~S}$ (441.17): C, 62.56; H, 6.16; N, 9.52; O, 14.49; S, 7.26. Found: $\mathrm{C}, 61.759$; $\mathrm{H}, 5.632 ; \mathrm{N}, 9$. 182. S, 6.911. ${ }^{1} \mathrm{H}$ NMR (DMSO-d $\left.{ }_{6}\right) \delta$ ppm: $0.89(3 \mathrm{H}, \mathrm{s}), 1.32-1.43(6 \mathrm{H}, \mathrm{d})$, $1.74(2 \mathrm{H}, \mathrm{s}), 2.35(3 \mathrm{H}, \mathrm{s}), 4.12(2 \mathrm{H}, \mathrm{s}), 7.151(2 \mathrm{H}, \mathrm{s}), 7.35(2 \mathrm{H}, \mathrm{s}), 7.82-7.89(3 \mathrm{H}, \mathrm{d}), 8.14$ $(2 \mathrm{H}, \mathrm{s}), 8.7(2 \mathrm{H}, \mathrm{s})$; IR $\mathrm{V}_{\max } \mathrm{cm}^{-1}(\mathrm{KBr}): 3350(\mathrm{NH}), 2997\left(\mathrm{CH}_{3}\right.$ stretch), 1737, (C=O ketone), 1643, $1587(\mathrm{C}=\mathrm{C}), 1157,1327(\mathrm{~S}=\mathrm{O}), 1406,\left(\mathrm{CH}_{3}\right.$ bend $), 1051(\mathrm{C}-\mathrm{O})$; Mass $(\mathrm{m} / \mathrm{e}): 441\left(\mathrm{M}^{+}\right)$.

4-(3-methyl-5-oxo-4-(3,4,5-trimethoxybenzylidene)-4,5-dihydro-1H-pyrazol-1-yl)benzene sulfonamide: (4b)

Crystallization: Ethanol (yield $85 \%$ ), colour: orange crystals. M.P.: $182-180{ }^{\circ} \mathrm{C}$. Anal. Calcd for $\mathrm{C}_{20} \mathrm{H}_{21} \mathrm{~N}_{3} \mathrm{O}_{6} \mathrm{~S}(431.12)$ : C, 55.67; H, 4.91; N, 9.74; O, 22.25; S, 7.43. Found: C, 49.359; $\mathrm{H}, 5.632 ; \mathrm{N}, 9.082 . \mathrm{S}, 10.091 .{ }^{1} \mathrm{H}$ NMR (DMSO-d 6$) \delta \mathrm{ppm}: 2.34(3 \mathrm{H}, \mathrm{s}), 3.88(9 \mathrm{H}, \mathrm{s}), 7.37$ $(2 \mathrm{H}, \mathrm{s}), 7.82-7.90(3 \mathrm{H}, \mathrm{d}), 8.11-8.20(4 \mathrm{H}, \mathrm{d})$; IR $\mathrm{V}_{\max } \mathrm{cm}^{-1}(\mathrm{KBr}): 3317(\mathrm{NH}), 3088$ (Aromatic $=\mathrm{CH}) 2976\left(\mathrm{CH}_{3}\right.$ stretch $), 1672,(\mathrm{C}=\mathrm{O}$ ketone $), 1500,1587(\mathrm{C}=\mathrm{C}), 1159,1330(\mathrm{~S}=\mathrm{O}), 1425$, $\left(\mathrm{CH}_{3}\right.$ bend), $1101(\mathrm{C}-\mathrm{O})$; Mass $(\mathrm{m} / \mathrm{e}): 431\left(\mathrm{M}^{+}\right)$.

4-(4-(2,5-dimethoxybenzylidene)-3-methyl-5-oxo-4,5-dihydro-1H-pyrazol-1-yl)benzenesulfon amide; (4c)

Crystallization: Ethanol (yield $80 \%$ ), colour: orange crystals. M.P.: $178-180{ }^{\circ} \mathrm{C}$. Anal. Calcd for $\mathrm{C}_{19} \mathrm{H}_{19} \mathrm{~N}_{3} \mathrm{O}_{5} \mathrm{~S}$ (401.10): C, 56.85; H, 4.77; N, 10.47; O, 19.93; S, 7.99. Found: C, 50.606; $\mathrm{H}, 4.707 ; \mathrm{N}, 11.841 . \mathrm{S}, 11.039 .{ }^{1} \mathrm{H}$ NMR (DMSO-d 6 ) $\delta$ ppm: $2.36(3 \mathrm{H}, \mathrm{s}), 3.369(6 \mathrm{H}, \mathrm{s}), 7.36$ $(2 \mathrm{H}, \mathrm{s}), 7.40(1 \mathrm{H}, \mathrm{s}), 7.88-7.91(2 \mathrm{H}, \mathrm{d}), 8.14-8.16(2 \mathrm{H}, \mathrm{d}), 8.25-8.30(3 \mathrm{H}, \mathrm{m})$; IR $\mathrm{V}_{\max } \mathrm{cm}^{-}$ ${ }^{1}(\mathrm{KBr}): 3288(\mathrm{NH}), 3078($ Aromatic $=\mathrm{CH}) 2833\left(\mathrm{CH}_{3}\right.$ stretch), 1681, $(\mathrm{C}=\mathrm{O}$ ketone), 1500, $1591(\mathrm{C}=\mathrm{C}), 1159,1323(\mathrm{~S}=\mathrm{O}), 1460,\left(\mathrm{CH}_{3}\right.$ bend $), 1095(\mathrm{C}-\mathrm{O})$; Mass $(\mathrm{m} / \mathrm{e}): 401\left(\mathrm{M}^{+}\right)$.

4-(4-(anthracen-9-ylmethylene)-3-methyl-5-oxo-4,5-dihydro-1H-pyrazol-1-yl)benzenesulfon amide; (4d)

Crystallization: Ethanol (yield $83 \%$ ), colour : Red crystals. M.P.: 176-178 ${ }^{\circ}$ C. Anal. Calcd for $\mathrm{C}_{25} \mathrm{H}_{19} \mathrm{~N}_{3} \mathrm{O}_{3} \mathrm{~S}$ (441.10): C, 68.01; H, 4.34; N, 9.52; O, 10.87; S, 7.26 Found: C, 66.914; $\mathrm{H}$, 5.149; N, 9.564. S, 9.620. ${ }^{1} \mathrm{H}$ NMR (DMSO-d 6 ) $\delta$ ppm: $1.24(3 \mathrm{H}, \mathrm{s}), 7.30-7.4(2 \mathrm{H}, \mathrm{d}), 7.58-$ 
$7.62(4 \mathrm{H}, \mathrm{dd}), 7.93(1 \mathrm{H}, \mathrm{s}), 8.14-8.16(2 \mathrm{H}, \mathrm{s}), 8.19-8.77(5 \mathrm{H}, \mathrm{m}), 8.81-8.88(2 \mathrm{H}, \mathrm{m})$; IR $\mathrm{V}_{\max }$ $\mathrm{cm}^{-1}(\mathrm{KBr}): 3345,3223(\mathrm{NH}), 3093($ Aromatic $=\mathrm{CH}) 2833\left(\mathrm{CH}_{3}\right.$ stretch), $1699(\mathrm{C}=\mathrm{O}$ ketone), 1618, $1591(\mathrm{C}=\mathrm{C}), 1155,1344(\mathrm{~S}=\mathrm{O}), 1496,\left(\mathrm{CH}_{3}\right.$ bend $)$, Mass $(\mathrm{m} / \mathrm{e}): 441\left(\mathrm{M}^{+}\right)$.

4-(4-(3,4-dimethoxybenzylidene)-3-methyl-5-oxo-4,5-dihydro-1H-pyrazol-1-yl)benzenesulfon amide; (4e)

Crystallization: Ethanol (yield $80 \%$ ), colour: red-orange crystals. M.P.: $180-182{ }^{\circ} \mathrm{C}$. Anal. Calcd for $\mathrm{C}_{19} \mathrm{H}_{19} \mathrm{~N}_{3} \mathrm{O}_{5} \mathrm{~S}$ (401.10): C, 56.85; H, 4.77; N, 10.47; O, 19.93; S, 7.99. Found: C, 51.609; H, 6.016; N, 8.777. S, 11.039. ${ }^{1} \mathrm{H}$ NMR (DMSO-d 6$) \delta$ ppm: $1.36(3 \mathrm{H}, \mathrm{s}), 3.48(6 \mathrm{H}, \mathrm{s})$, $7.32(2 \mathrm{H}, \mathrm{s}), 7.38(1 \mathrm{H}, \mathrm{s}), 7.80-7.86(2 \mathrm{H}, \mathrm{d}), 8.12-8.14(2 \mathrm{H}, \mathrm{d}), 8.28-8.39(3 \mathrm{H}, \mathrm{m}) ; \mathrm{IR} \mathrm{V}_{\max } \mathrm{cm}^{-1}$ $(\mathrm{KBr}): 3284(\mathrm{NH}), 3097($ Aromatic $=\mathrm{CH})$ 1693, $(\mathrm{C}=\mathrm{O}$ ketone $), 1552,1597(\mathrm{C}=\mathrm{C}), 1155,1330$ $(\mathrm{S}=\mathrm{O}), 1095(\mathrm{C}-\mathrm{O})$; Mass $(\mathrm{m} / \mathrm{e}): 401\left(\mathrm{M}^{+}\right)$.

\section{4-(3-methyl-5-oxo-4-(thiophen-2-ylmethylene)-4,5-dihydro-1H-pyrazol-1-yl)benzenesulfona} mide; (4f)

Crystallization: Ethanol (yield $84 \%$ ), colour: yellow crystals. M.P.: 172-174 ${ }^{\circ} \mathrm{C}$. Anal. Calcd for $\mathrm{C}_{15} \mathrm{H}_{13} \mathrm{~N}_{3} \mathrm{O}_{3} \mathrm{~S}_{2}$ (347.040): C, 51.86; H, 3.77; N, 12.10; O, 13.82; S, 18.46. Found: $\mathrm{C}$, 51.911; H, 4.718; N, 12.218. S, 20.694. ${ }^{1} \mathrm{H}$ NMR (DMSO-d 6 ) $\delta$ ppm: $2.36(3 \mathrm{H}, \mathrm{s}), 7.36-7.40$ $(3 \mathrm{H}, \mathrm{d}), 7.89-7.91(2 \mathrm{H}, \mathrm{d}), 8.14-8.16(2 \mathrm{H}, \mathrm{d}), 8.25-8.29(3 \mathrm{H}, \mathrm{m}) ; \mathrm{IR} \mathrm{V}_{\max } \mathrm{cm}^{-1}(\mathrm{KBr}): 3284$ $(\mathrm{NH}), 3097($ Aromatic $=\mathrm{CH})$ 1693, $(\mathrm{C}=\mathrm{O}$ ketone), 1552, $1597(\mathrm{C}=\mathrm{C}), 1155,1330(\mathrm{~S}=\mathrm{O})$, 1095 (C-O); Mass (m/e): $347\left(\mathrm{M}^{+}\right)$.

\section{4-(4-(4-chlorobenzylidene)-3-methyl-5-oxo-4,5-dihydro-1H-pyrazol-1-} yl)benzenesulfonamide; (4g)

Crystallization: Ethanol (yield $76 \%$ ), colour: yellow crystals. M.P.: 194-192 ${ }^{\circ} \mathrm{C}$. Anal. Calcd for $\mathrm{C}_{17} \mathrm{H}_{14} \mathrm{ClN}_{3} \mathrm{O}_{3} \mathrm{~S}$ (375.044): $\mathrm{C}, 54.33 ; \mathrm{H}, 3.75 ; \mathrm{Cl}, 9.43 ; \mathrm{N}, 11.18 ; \mathrm{O}, 12.77 ; \mathrm{S}, 8.53$. Found: C, 53.911; H, 4.118; N, 11.218. S, 9.694. ${ }^{1} \mathrm{H}$ NMR (DMSO-d 6 ) $\delta$ ppm: $2.12(3 \mathrm{H}, \mathrm{s})$, $7.33(2 \mathrm{H}, \mathrm{s}), 7.75-7.82(3 \mathrm{H}, \mathrm{d}), 8.25-8.39(6 \mathrm{H}, \mathrm{m}) ; \mathrm{IR} \mathrm{V}_{\max } \mathrm{cm}^{-1}(\mathrm{KBr}): 3279(\mathrm{NH}), 3080$ $($ Aromatic $=\mathrm{CH})$ 1707, $(\mathrm{C}=\mathrm{O}$ ketone $), 1487,1593(\mathrm{C}=\mathrm{C}), 1155,1335(\mathrm{~S}=\mathrm{O})$; Mass $(\mathrm{m} / \mathrm{e}): 375$ $\left(\mathrm{M}^{+}\right)$.

\section{4-(3-methyl-4-(4-methylbenzylidene)-5-oxo-4,5-dihydro-1H-pyrazol-1-} yl)benzenesulfonamide; (4h)

Crystallization: Ethanol (yield $79 \%$ ), colour: yellow crystals. M.P.: $168-166^{\circ} \mathrm{C}$. Anal. Calcd for $\mathrm{C}_{18} \mathrm{H}_{17} \mathrm{~N}_{3} \mathrm{O}_{3} \mathrm{~S}$ (355.02): C, 60.83; H, 4.82; N, 11.82; O, 13.50; S, 9.02. Found: C, 59.911; $\mathrm{H}, 4.781$; N, 12.218. S, 8.694. ${ }^{1} \mathrm{H}$ NMR $\left(\mathrm{DMSO}_{-} \mathrm{d}_{6}\right) \delta \mathrm{ppm}: 2.15(3 \mathrm{H}, \mathrm{s}), 6.99(3 \mathrm{H}, \mathrm{s}), 7.12$ (2H,s), 7.14-7.24 (2H,m), 7.68-8.2(6H,m); IR V $\mathrm{max} \mathrm{cm}^{-1}(\mathrm{KBr}): 3285(\mathrm{NH}), 3086$ (Aromatic $=\mathrm{CH})$ 1772, $(\mathrm{C}=\mathrm{O}$ ketone $), 1494,1593(\mathrm{C}=\mathrm{C}), 1159,1329(\mathrm{~S}=\mathrm{O})$; Mass $(\mathrm{m} / \mathrm{e}): 375\left(\mathrm{M}^{+}\right)$.

\section{4-(3-methyl-4-(naphthalen-1-ylmethylene)-5-oxo-4,5-dihydro-1H-pyrazol-1-yl)benzenesul} Fonamide; (4i)

Crystallization: Ethanol (yield $76 \%$ ), colour: yellow crystals. M.P.: $186-188{ }^{\circ} \mathrm{C}$. Anal. Calcd for $\mathrm{C}_{21} \mathrm{H}_{17} \mathrm{~N}_{3} \mathrm{O}_{3} \mathrm{~S}$ (391.10): C, 64.43; H, 4.38; N, 10.73; O, 12.26; S, 8.19. Found: C, 62.45; $\mathrm{H}, 4.118 ; \mathrm{N}, 11.20 . \mathrm{S}, 8.60 .{ }^{1} \mathrm{H}$ NMR $\left(\mathrm{DMSO}_{-} \mathrm{d}_{6}\right) \delta \mathrm{ppm}: 1.28(3 \mathrm{H}, \mathrm{s}), 7.28-7.4(2 \mathrm{H}, \mathrm{d}), 7.48-$ $7.52(4 \mathrm{H}, \mathrm{m}), 8.11-8.14(2 \mathrm{H}, \mathrm{s}), 8.39-8.48(6 \mathrm{H}, \mathrm{m}) ; \mathrm{IR} \mathrm{V}_{\max } \mathrm{cm}^{-1}(\mathrm{KBr}): 3254(\mathrm{NH}), 3186$ $($ Aromatic $=\mathrm{CH})$ 1674, $(\mathrm{C}=\mathrm{O}$ ketone $), 1494,1595(\mathrm{C}=\mathrm{C}), 1159,1325(\mathrm{~S}=\mathrm{O})$; Mass $(\mathrm{m} / \mathrm{e})$ : 391 $\left(\mathrm{M}^{+}\right)$. 
4-(3-methyl-4-(4-nitrobenzylidene)-5-oxo-4,5-dihydro-1H-pyrazol-1yl)benzenesulfonamide; $(4 \mathrm{j})$

Crystallization: Ethanol (yield $68 \%$ ), colour: dark brown crystals. M.P.: $170-172{ }^{\circ} \mathrm{C}$. Anal. Calcd for $\mathrm{C}_{17} \mathrm{H}_{14} \mathrm{~N}_{4} \mathrm{O}_{5} \mathrm{~S}$ (386.068): C, 52.84; H, 3.65; N, 14.50; O, 20.70; S, 8.30. Found: C, 50.75; H, 4.00; N, 15.68. S, 9.50. ${ }^{1} \mathrm{H}$ NMR (DMSO-d $\left.\mathrm{d}_{6}\right) \delta \mathrm{ppm}: 2.18(3 \mathrm{H}, \mathrm{s}), 7.28(2 \mathrm{H}, \mathrm{s})$, 7.78-7.91 (3H, m), 8.40-8.69 (6H,m); IR V $\mathrm{max}_{\max } \mathrm{cm}^{-1}(\mathrm{KBr}): 3342(\mathrm{NH}), 3211($ Aromatic $=\mathrm{CH})$ 1691, $(\mathrm{C}=\mathrm{O}$ ketone $), 1487,1591(\mathrm{C}=\mathrm{C}), 1159,1342(\mathrm{~S}=\mathrm{O})$; Mass $(\mathrm{m} / \mathrm{e}): 386 .\left(\mathrm{M}^{+}\right)$.

4-(4-(3-methoxybenzylidene)-3-methyl-5-oxo-4,5-dihydro-1H-pyrazol-1-yl)benzenesulfon amide; (4k)

Crystallization: Ethanol (yield $80 \%$ ), colour: orange crystals. M.P.: 252-250 ${ }^{\circ}$ C. Anal. Calcd for $\mathrm{C}_{18} \mathrm{H}_{17} \mathrm{~N}_{3} \mathrm{O}_{4} \mathrm{~S}$ (371.09): C, 58.21; H, 4.61; N, 11.31; O, 17.23; S, 8.63 Found: C, 55.45; H, 5.24; N, 12.45. S, 10.24. ${ }^{1} \mathrm{H}$ NMR (DMSO-d 6$) \delta$ ppm: $2.32(3 \mathrm{H}, \mathrm{s}), 3.4(3 \mathrm{H}, \mathrm{s}), 6.77(2 \mathrm{H}, \mathrm{s})$, 7.11-7.39 $(4 \mathrm{H}, \mathrm{m}), 7.52-8.12(5 \mathrm{H}, \mathrm{m})$; IR V $\mathrm{max}_{\max } \mathrm{cm}^{-1}(\mathrm{KBr}): 3257(\mathrm{NH}), 3078($ Aromatic $=\mathrm{CH})$ $2922\left(\mathrm{CH}_{3}\right.$ stretch $), 1680,(\mathrm{C}=\mathrm{O}$ ketone $), 1595(\mathrm{C}=\mathrm{C}), 1159,1332(\mathrm{~S}=\mathrm{O}), 1460,\left(\mathrm{CH}_{3}\right.$ bend $)$, 1037 (C-O); Mass (m/e) :371( $\left.\mathrm{M}^{+}\right)$.

4-(4-(4-methoxybenzylidene)-3-methyl-5-0xo-4,5-dihydro-1H-pyrazol-1-yl)benzenesulfon amide; (4l)

Crystallization: Ethanol (yield $74 \%$ ), colour: orange crystals. M.P.: $168-170{ }^{\circ} \mathrm{C}$. Anal. Calcd for $\mathrm{C}_{18} \mathrm{H}_{17} \mathrm{~N}_{3} \mathrm{O}_{4} \mathrm{~S}$ (371.09): C, 58.21; H, 4.61; N, 11.31; O, 17.23; S, 8.63 Found: C, 58.00; H, 4.24; N, 12.00. S, 9.24. ${ }^{1} \mathrm{H}$ NMR (DMSO-d 6$) \delta \mathrm{ppm}: 2.35(3 \mathrm{H}, \mathrm{s}), 3.86(3 \mathrm{H}, \mathrm{s}), 6.78-6.80$ $(2 \mathrm{H}, \mathrm{d}), 7.14-7.16(2 \mathrm{H}, \mathrm{d}), 7.36-7.88(4 \mathrm{H}, \mathrm{m}), 8.13-8.72(3 \mathrm{H}, \mathrm{m}) ; \mathrm{IR} \mathrm{V}_{\max } \mathrm{cm}^{-1}(\mathrm{KBr}): 3341$ $(\mathrm{NH}), 3261($ Aromatic $=\mathrm{CH}) 2922\left(\mathrm{CH}_{3}\right.$ stretch $), 1676,(\mathrm{C}=\mathrm{O}$ ketone $), 1577(\mathrm{C}=\mathrm{C}), 1155$, $1327(\mathrm{~S}=\mathrm{O}), 1460,\left(\mathrm{CH}_{3}\right.$ bend $), 1030(\mathrm{C}-\mathrm{O})$; Mass $(\mathrm{m} / \mathrm{e}): 371\left(\mathrm{M}^{+}\right)$.

4-(4-(3-bromo-4-methoxybenzylidene)-3-methyl-5-oxo-4,5-dihydro-1H-pyrazol-1-1)benzene Sulfonamide; $(4 \mathrm{~m})$

Crystallization: Ethanol (yield $83 \%$ ), colour: brown crystals. M.P.: $182-180{ }^{\circ} \mathrm{C}$. Anal. Calcd for $\mathrm{C}_{18} \mathrm{H}_{16} \mathrm{BrN}_{3} \mathrm{O}_{4} \mathrm{~S}$ (449.00): C, 48.01; H, 3.58; $\mathrm{Br}, 17.74 ; \mathrm{N}, 9.33 ; \mathrm{O}, 14.21 ; \mathrm{S}, 7.12$. Found: C, 55.45; H, 5.24; N, 12.45. S, 10.24. ${ }^{1} \mathrm{H}$ NMR (DMSO-d 6 ) $\delta$ ppm: $2.34(3 \mathrm{H}, \mathrm{s}), 2.89(3 \mathrm{H}, \mathrm{s})$, 7.02-7.04 $(2 \mathrm{H}, \mathrm{d}), 7.12-7.16(3 \mathrm{H}, \mathrm{m}), 7.66-7.89(3 \mathrm{H}, \mathrm{m}), 7.92-8.12(2 \mathrm{H}, \mathrm{m}) ; \mathrm{IR} \mathrm{V}_{\max } \mathrm{cm}^{-1}$ $(\mathrm{KBr}): 3252(\mathrm{NH}), 3078($ Aromatic $=\mathrm{CH}) 2922\left(\mathrm{CH}_{3}\right.$ stretch), 1666, $(\mathrm{C}=\mathrm{O}$ ketone), 1589 $(\mathrm{C}=\mathrm{C}), 1157,1332(\mathrm{~S}=\mathrm{O}), 1460,\left(\mathrm{CH}_{3}\right.$ bend $), 1099(\mathrm{C}-\mathrm{O})$; Mass $(\mathrm{m} / \mathrm{e}): 449\left(\mathrm{M}^{+}\right)$.

Table 1. Optimization of (\%) yield for the microwave assisted synthesis of $4 \mathrm{a}, 4 \mathrm{~b}, 4 \mathrm{c}$ and $4 \mathrm{e}$ using different solvents and catalyst.

\begin{tabular}{|c|c|c|c|c|}
\hline Entry as & Solvent & catalyst & $\begin{array}{l}\text { Time }^{\mathrm{a}} \text { (min) } \\
\text { microwave }\end{array}$ & Yield $^{\mathrm{b}} \%$ \\
\hline \multirow{2}{*}{$4 a$} & \multirow{2}{*}{ Methanol } & Piperidine & 10 & 88 \\
\hline & & TEA & 15 & 75 \\
\hline \multirow{2}{*}{$4 \mathbf{a}$} & \multirow{2}{*}{ Chloroform } & Piperidine & 18 & 80 \\
\hline & & TEA & 25 & 74 \\
\hline
\end{tabular}




\begin{tabular}{|c|c|c|c|c|}
\hline \multirow{2}{*}{$4 a$} & \multirow{2}{*}{ Ethyl Acetate } & Piperidine & 35 & 68 \\
\hline & & TEA & 38 & 55 \\
\hline \multirow{2}{*}{$4 a$} & \multirow{2}{*}{ DMF } & $\mathrm{K}_{2} \mathrm{CO}_{3}$ & 8 & 65 \\
\hline & & $\mathrm{C}_{\mathrm{S}} \mathrm{CO}_{3}$ & 8 & 68 \\
\hline $4 a$ & Methanol & $\mathrm{HCl}$ & 30 & 48 \\
\hline $4 a$ & Acetic acid & - & 25 & 45 \\
\hline \multirow{2}{*}{$4 b$} & \multirow{2}{*}{ Methanol } & Piperidine & 8 & 92 \\
\hline & & TEA & 10 & 85 \\
\hline \multirow{2}{*}{$4 b$} & \multirow{2}{*}{ Chloroform } & Piperidine & 15 & 82 \\
\hline & & TEA & 18 & 76 \\
\hline \multirow{2}{*}{$4 b$} & \multirow{2}{*}{ Ethyl Acetate } & Piperidine & 20 & 70 \\
\hline & & TEA & 22 & 69 \\
\hline \multirow{2}{*}{$4 b$} & \multirow{2}{*}{$\mathrm{DMF}$} & $\mathrm{K}_{2} \mathrm{CO}_{3}$ & 5 & 69 \\
\hline & & $\mathrm{C}_{\mathrm{S}} \mathrm{CO}_{3}$ & 5 & 68 \\
\hline $4 b$ & Methanol & $\mathrm{HCl}$ & 28 & 56 \\
\hline $4 b$ & Acetic acid & - & 30 & 52 \\
\hline \multirow{2}{*}{$4 c$} & \multirow{2}{*}{ Methanol } & Piperidine & 12 & 90 \\
\hline & & TEA & 10 & 88 \\
\hline \multirow{2}{*}{$4 c$} & \multirow{2}{*}{ Chloroform } & Piperidine & 18 & 86 \\
\hline & & TEA & 15 & 80 \\
\hline \multirow{2}{*}{$4 c$} & \multirow{2}{*}{ Ethyl Acetate } & Piperidine & 20 & 74 \\
\hline & & TEA & 20 & 72 \\
\hline \multirow{2}{*}{$4 c$} & \multirow{2}{*}{ DMF } & $\mathrm{K}_{2} \mathrm{CO}_{3}$ & 6 & 68 \\
\hline & & $\mathrm{C}_{\mathrm{S}} \mathrm{CO}_{3}$ & 6 & 64 \\
\hline $4 c$ & Methanol & $\mathrm{HCl}$ & 25 & 50 \\
\hline $4 c$ & Acetic acid & - & 30 & 54 \\
\hline \multirow{2}{*}{$4 e$} & \multirow{2}{*}{ Methanol } & Piperidine & 8 & 79 \\
\hline & & TEA & 12 & 72 \\
\hline
\end{tabular}




\begin{tabular}{|c|c|c|c|c|}
\hline \multirow{2}{*}{$4 \mathbf{e}$} & \multirow{2}{*}{ Chloroform } & Piperidine & 14 & 70 \\
\cline { 3 - 5 } & & TEA & 18 & 64 \\
\hline \multirow{2}{*}{$4 \mathbf{4}$} & Ethyl Acetate & Piperidine & 25 & 58 \\
\cline { 3 - 5 } & & $\mathrm{TEA}$ & 28 & 54 \\
\hline \multirow{2}{*}{$4 \mathbf{4}$} & \multirow{2}{*}{$\mathrm{DMF}$} & $\mathrm{K}_{2} \mathrm{CO}_{3}$ & 6 & 54 \\
\cline { 3 - 5 } & & $\mathrm{C}_{\mathrm{S}} \mathrm{CO}_{3}$ & 6 & 52 \\
\hline \multirow{2}{*}{$4 \mathbf{4}$} & Methanol & $\mathrm{HCl}$ & 30 & 48 \\
\hline \multirow{4}{*}{$4 \mathbf{e}$} & Acetic acid & - & 25 & 45 \\
\hline
\end{tabular}

Table 2. Comparison of yield (\%) of the Arylidene derivatives obtained using microwave assisted as well as conventional method of synthesis.

\begin{tabular}{|c|c|c|}
\hline \multirow[b]{2}{*}{ compound } & \multicolumn{2}{|c|}{ Yield (\%) obtained by the method of synthesis } \\
\hline & $\begin{array}{c}\begin{array}{c}\text { Microwave assisted } \\
\text { method }\end{array} \\
\end{array}$ & $\begin{array}{c}\text { Conventional } \\
\text { method }\end{array}$ \\
\hline $4 a$ & 88 & 74 \\
\hline $4 b$ & 92 & 75 \\
\hline $4 c$ & 90 & 78 \\
\hline $4 d$ & 82 & 62 \\
\hline $4 e$ & 88 & 76 \\
\hline $4 f$ & 79 & 63 \\
\hline $4 g$ & 78 & 68 \\
\hline $4 h$ & 70 & 48 \\
\hline $4 i$ & 78 & 63 \\
\hline $4 j$ & 77 & 54 \\
\hline $4 k$ & 85 & 66 \\
\hline 41 & 86 & 62 \\
\hline $4 m$ & 80 & 68 \\
\hline
\end{tabular}


Table 3. Physical data of the pyrazolone derivatives obtained using microwave assisted method of synthesis $4(\mathrm{a}-\mathrm{m})$.

\begin{tabular}{|c|c|c|c|c|c|c|}
\hline Compounds & Substitution $\mathbf{R}$ & $\begin{array}{c}\text { Molecular } \\
\text { formula }\end{array}$ & $\begin{array}{c}\text { Molecular } \\
\text { weight }\end{array}$ & Color & $\begin{array}{l}\text { Yield } \\
(\%)\end{array}$ & $\begin{array}{c}\text { M.P. } \\
{ }^{\circ} \mathrm{C}\end{array}$ \\
\hline $4 a$ & $-\mathrm{OC}_{6} \mathrm{H}_{13}$ & $\mathrm{C}_{23} \mathrm{H}_{27} \mathrm{~N}_{3} \mathrm{O}_{4} \mathrm{~S}$ & 441.17 & yellow & $87 \%$ & $160-162$ \\
\hline $4 b$ & & $\mathrm{C}_{20} \mathrm{H}_{21} \mathrm{~N}_{3} \mathrm{O}_{6} \mathrm{~S}$ & 431.12 & orange & $85 \%$ & $182-180$ \\
\hline $4 c$ & & $\mathrm{C}_{19} \mathrm{H}_{19} \mathrm{~N}_{3} \mathrm{O}_{5} \mathrm{~S}$ & 401.10 & orange & $80 \%$ & $178-180$ \\
\hline 4d & & $\mathrm{C}_{25} \mathrm{H}_{19} \mathrm{~N}_{3} \mathrm{O}_{3} \mathrm{~S}$ & 441.10 & Red & $83 \%$ & $176-178$ \\
\hline $4 e$ & & $\mathrm{C}_{19} \mathrm{H}_{19} \mathrm{~N}_{3} \mathrm{O}_{5} \mathrm{~S}$ & 401.10 & $\begin{array}{c}\text { red- } \\
\text { orange }\end{array}$ & $80 \%$ & $180-182$ \\
\hline $4 f$ & & $\mathrm{C}_{15} \mathrm{H}_{13} \mathrm{~N}_{3} \mathrm{O}_{3} \mathrm{~S}_{2}$ & 347.04 & Yellow & $84 \%$ & $172-174$ \\
\hline $4 g$ & & $\mathrm{C}_{17} \mathrm{H}_{14} \mathrm{ClN}_{3} \mathrm{O}_{3} \mathrm{~S}$ & 375.04 & Yellow & $76 \%$ & 194-192 \\
\hline $4 h$ & & $\mathrm{C}_{18} \mathrm{H}_{17} \mathrm{~N}_{3} \mathrm{O}_{3} \mathrm{~S}$ & 355.02 & Yellow & $79 \%$ & $168-166$ \\
\hline $4 i$ & & $\mathrm{C}_{21} \mathrm{H}_{17} \mathrm{~N}_{3} \mathrm{O}_{3} \mathrm{~S}$ & 391.10 & Yellow & $76 \%$ & $186-188$ \\
\hline $4 j$ & & $\mathrm{C}_{17} \mathrm{H}_{14} \mathrm{~N}_{4} \mathrm{O}_{5} \mathrm{~S}$ & 386.06 & $\begin{array}{l}\text { dark } \\
\text { brown }\end{array}$ & $68 \%$ & $252-250$ \\
\hline $4 k$ & & $\mathrm{C}_{18} \mathrm{H}_{17} \mathrm{~N}_{3} \mathrm{O}_{4} \mathrm{~S}$ & 371.09 & Orange & $80 \%$ & $174-176$ \\
\hline
\end{tabular}




\begin{tabular}{|l|l|l|l|l|l|l|}
\hline $\mathbf{4 1}$ & & $\mathrm{C}_{18} \mathrm{H}_{17} \mathrm{~N}_{3} \mathrm{O}_{4} \mathrm{~S}$ & 371.09 & Orange & $74 \%$ & $168-170$ \\
\hline $\mathbf{4 m}$ & & $-\mathrm{OCH}_{3}$ & & & & \\
\hline
\end{tabular}

We have synthesized 4a, 4b, 4c, and $4 \mathrm{e}$ in diverse solvents like Methanol, chloroform, Ethyl Acetate, and DMF by using different basic catalyst such as piperidine, TEA as an organic base and $\mathrm{K}_{2} \mathrm{CO}_{3}, \mathrm{C}_{\mathrm{S}} \mathrm{CO}_{3}$ as an inorganic base under microwave irradiation. Synthesis was also carried out in acidic media by using methanol as solvent, few drops of $\mathrm{HCl}$ as catalyst. We have also use gl. Acetic acid for the optimisation of yield. We observed that reaction carried out in DMF and inorganic base taken lesser time but yield observed very low, but in case of methanol as solvent and piperidine as basic organic catalyst, instead of, chloroform, DMF and Ethyl Acetate as solvents and other acidic or basic catalyst. The time taken for the completion of reaction was 3-9 minutes and the \% yields observed was $85-90 \%$ under microwave assisted method. Furthermore, we carried out the same synthesis by using methanol as solvent and piperidine as catalyst under conventional method. The time taken for synthesis of compounds 4a-m by conventional method was around 6-8 hours and the \% yield observed was about $60-75 \%$. All the synthesized compounds were characterized by TLC, Melting point, elemental analysis, IR ${ }^{1} \mathrm{H}$ NMR.

\section{CONCLUSION}

Novel Sulphonamide Arylidene derivatives were synthesized in rationally good yields by microwave assisted method. This is environmentally compassionate technique gives higher fraction of yields and lesser reaction time and easy work up method.

\section{ACKNOWLEDGEMENTS}

The authors are thankful to Department of Chemistry, Saurashtra University, Rajkot and specially indebted to "National Facility for Drug Discovery through New Chemical Entities (NCE's), Development \& Instrumentation Support to Small Manufacturing Pharma Enterprises", a programme under Drug \& Pharma Research Support (DPRS) jointly funded by Department of Science \& Technology, New Delhi, Government of Gujarat (Industries Commissioner ate) \& Saurashtra University, Rajkot.

\section{References}

[1] Abdel-Aziz M., Abuo-Rahma G. E. A., Hasan A. A., Eur. J. Med. Chem. 44 (2009) 3480-3487.

[2] Brune M. D. K., Acute Pain 1(1) (1997) 33-40.

[3] Das N., Verma A., Strivastava P. K., Shrivastava S. K., Ind. J. Chem. 47B (2008) 1555-1558. 
[4] Georgewill O. A, Georgewill U. O., Nwankwoala, R. N. P., The International. J. of Pharmacol. 7(1) (2009) 220-225.

[5] Kees K. L., Fitzgerald J. J., Steiner K. E., Mattes J. F., Mihan B., Tosi T., Mondoro D., McCaleb M. L., J. Med. Chem. 1996, 39 (1996) 3920-3928.

[6] Knorr L., Chem. Ber. 17 (1883) 546-552.

[7] Mariappan G., Saha B. P, Sutharson L, Ankits G., Pandey L., Kumar D., Journal of Pharmacy Research 3(12) (2010) 2856-2859.

[8] Maywald V., Steinmetz A., Rack M., Gotz N., Gotz R., Henkelmann J., Becker H., Synthesis of pyrazolones. Aiscar Bayeto, PCT Int. Appl. WO 0031042 A2, 2000, (Chem. Abstr., 133, 4655).

[9] M. Arnost, A. Pierce, E. Haar, D. Lauffer, J. Madden, K. Tanner, J. Green, Bioorg. Med. Chem. Lett., 20 (2010) 1661.

[10] R. Venkat Ragavan, V. Vijaykumar, N. Suchetha Kumari, Eur. J. Med. Chem. 44 (2009) 3852.

[11] A. Gursoy, S. Demirayak, G. Capan, K. Erol, K. Vural, Eur. J. Med. Chem. 35 (2000) 359.

[12] D. Castagnolo, F. Manetti, M. Radi, B. Bechi, M. Pagano, A. D. Logu, R. Meleddu, M. Saddi, M. Botta, Bioorg. Med. Chem. 17 (2009) 5716.

[13] Nikhil Parekh, Kalpana Maheria, Pratik Patel, Manoj Rathod, Int. J. PharmTech Res. 3 (2011) 540.

[14] G. Mariappan, B.P. Saha, L. Sutharson, A. Haldar, Indian J. Chem. 49B (2010) 1671.

[15] P. Manojkumar, T. K. Ravi, G. Subbuchettiar, Acta Pharm. 59 (2009) 159.

[16] A. M. Isloor, B. Kalluraya, P. Shetty, Eur. J. Med. Chem. 44 (2009) 3784.

[17] A. M. Isloor, B. Kalluraya, M. Rao, J. Saudi Chem. Soc. 4 (2000) 265.

[18] B. Kalluraya, A.M. Isloor, P.V. Frank, R.L. Jagadesha, S. Shenoy, Indian J. Heterocycl. Chem. 4 (2001) 159.

[19] D. Sunil, A.M. Isloor, P. Shetty, Der Pharma Chemica 1 (209) 19.

[20] A.M. Isloor, B. Kalluraya, K. S. Pai, Eur. J. Med. Chem. 45 (2010) 825.

[21] B. Chandrakantha, P. Shetty, V. Nambiyar, N. Isloor, A.M. Isloor, Eur. J. Med. Chem. 45 (2010) 1206.

[22] U. S. Rai, A. M. Isloor, P. Shetty, A. M. Vijesh, N. Prabhu, S. Isloor, M. Thiageeswaran, H. K. Fun, Eur. J. Med. Chem. 45 (2010) 2695.

[23] A. M. Vijesh, A. M. Isloor, V. Prabhu, S. Ahmad, S. Malladi, Eur. J. Med. Chem. 45 (2010) 5460 .

[24] V. Kumar, A. K. Abbas, N. Fausto, R. N. Mitchell, In Robbins Basic Pathology. $8^{\text {th }}$ Ed. (Reed Elsevier IndPvt. Ltd. India, 2007) 31. 